\title{
Beobachtungen über das Drehungsvermögen weinsaurer Salze
}

von

\section{Richard Přibram.}

Aus dem chemischen Laboratorium der $\mathrm{k}$. k. Universität in Czernowitz.

(Vorgelegt in der Sitzung am 16. November 1893.)

Guye ${ }^{1}$ hat bekanntlich die Ansicht ausgesprochen, dass der Zahlenwerth für das Drehvermögen optisch activer Körper von der Grösse oder dem Moleculargewichte der an eine active Substanz gebundenen Gruppen abhängig sei. So zeigt sich z. B. bei den Estern der Weinsäure eine Zurahme des Drehungsvermögens, wenn man die Wasserstoffe der beiden $\mathrm{COOH}$ Gruppen allmälig der Reihe nach durch die Gruppen $\mathrm{CH}_{3}$, $\mathrm{C}_{2} \mathrm{H}_{5}, \mathrm{C}_{3} \mathrm{H}_{7}, \mathrm{C}_{4} \mathrm{H}_{9} \ldots$ ersetzt. Anderseits fand Landolt ${ }^{2}$ bei Prüfung des Mulder-Krecke'schen Gesetzes der multiplen Drehungen, dass wässerige Lösungen verschiedener weinsaurer Salze von gleichem Weinsäuregehalt nahezu gleich stark drehen und dass die Verschiedenheit des Metalls einen verhältnissmässig nur geringen Einfluss auf die Mol. Rot. ausübt, welcher in keiner Beziehung zu dem Atomgewicht zu stehen scheint, und Oudemanns ${ }^{3}$ stellte die Regel auf, dass das moleculare Drehungsvermögen der Salze in etwas verdünnterer Lösung unabhängig von der Beschaffenheit des nicht activen Theiles ist.

1 Étude sur la dissymétrie moléculaire. Thèse Paris 1891 und Archives des sciences physiques et naturelles Genève; 3 période, tome 26.

2 Berichte d. deutsch. chem. Gesellsch. 1873, 2, 1073.

3 Versl. en Mededeel d. kon. Ak. van Wetensch. Amsterdam, (3), 3, 408. 
Seither haben sich aus den Untersuchungen, welche Sonnenthal ${ }^{1}$ in meinem Laboratorium durchgeführt hat, Andeutungen ergeben, aus welchen hervorgeht, dass die Grösse des Atomgewichtes des in einem weinsauren Salz vorhandenen Metalles auf die Grösse der Drehung der Salzlösung doch einen Einfluss zu üben vermag.

Sonnenthal hatte nur Kalium-, Natrium-, Lithium- und Ammoniumsalze in sehr verdünnten Lösungen untersucht. Ich habe nun Herrn cand. phil. We chsler veranlasst, die Sonnenthal'schen Versuche zu wiederholen und auf andere Salze auszudehnen. Es schien mir von Bedeutung, namentlich Metalle mit höherem Atomgewicht in Betracht zu ziehen, weil bei denselben ein etwaiger Einfluss besonders deutlich hervortreten musste. Hiebei wurden insbesondere bei der Untersuchung der Thalliumsalze bemerkenswerthe Resultate erhalten. Ich hatte ursprünglich die Absicht mit der Veröffentlichung der diesbezüglichen Studien bis zum völligen Abschluss derselben zu zögern.

Inzwischen ist jedoch eine Arbeit von $\mathrm{H}$ ä d rich ${ }^{2}$ erschienen, welche allerdings auf Grundlage eines ganz anderen Arbeitsmateriales zum Theil zu ähnlichen Ergebnissen führte, wie diejenigen, welche in meinem Laboratorium erhalten wurden. Ich will deshalb mit einer vorläufigen Mittheilung der bisher gewonnenen Ergebnisse nicht zurückhalten, da namentlich die Untersuchung der Thalliumsalze zu Resultaten geführt hat, die ein weiteres Studium erheischen, das ich mir gerne vorbehalten möchte.

Hädrich untersuchte eine Anzahl von Alkaloidsalzen und seine Messungen stimmen in bemerkenswerther Weise mit dem früher erwähnten Oudemann'schen Gesetz.

Die von Herrn Wechs1er vorgenommenen Bestimmungen erstrecken sich auf die neutralen weinsauren Salze des Lithium, Ammonium, Natrium, Kalium, Rubidium, Caesium und Thallium sowie auf die sauren Salze des Natrium, Kalium, Rubidium, Caesium und Thallium.

1 Sitzungsber. der kaiserl. Akad. der Wissensch. Wien, mathem.-naturw. C1. Bd. 100, November 1891.

2 Zeitschrift für physikal. Chemie, 12, 476. 
Es sind das so ziemlich alle Metallsalze der Weinsäure, welche mit Rücksicht auf die Löslichkeit überhaupt in Betracht gezogen werden konnten.

Die Lösungen wurden in der Art bereitet, dass in $100 \mathrm{~cm}^{3}$ Wasser stets das der Menge $0.2 g$ Weinsäure äquivalente Gewicht des Tartrates aufgelöst wurde. Die polarimetrischen Beobachtungen wurden mit einem Landolt-Lippich'schen Halbschattenapparate vorgenommen, demselben Apparate, den ich bereits bei früheren Untersuchungen benützte und der auch bei den Versuchen von Soninenthal zur Verwendung gelangte. Auch die Versuchsbedingungen waren die gleichen. Jede Lösung wurde einige Male innerhalb gewisser Zeitintervalle beobachtet, und zwar so lange, bis zwei in verschiedenen Zeiträumen wiederholte Beobachtungen übereinstimmende Resultate ergaben, somit angenommen werden konnte, dass die für die betreffende Concentration weitest gehende Dissociation erreicht war. Der erhaltene Werth wurde als der für die Betrachtungen massgebende Endwerth erachtet. Jedes Salz wurde zur Feststellung der Reinheit vor der Verwendung untersucht. Ausführlicheres hierüber wird in einer späteren Abhandlung mitgetheilt werden.

In der nachfolgenden Tabelle bedeutet:

$p=$ Gewichtsprocent der Lösung an activer Substanz.

$d=$ specifisches Gewicht der Lösung bei $20^{\circ}$.

$\sigma_{D}^{20}=$ beobachteter Drehungswinkel bei der Temperatur $20^{\circ}$ unter Anwendung von Natriumlicht und eines Beob. achtungsrohres von 3 dm Länge.

$[\alpha]_{D}=$ berechnete specifische Drehung.

$[M]_{D}=$ Moleculare Drehung. 
Tabelle I.

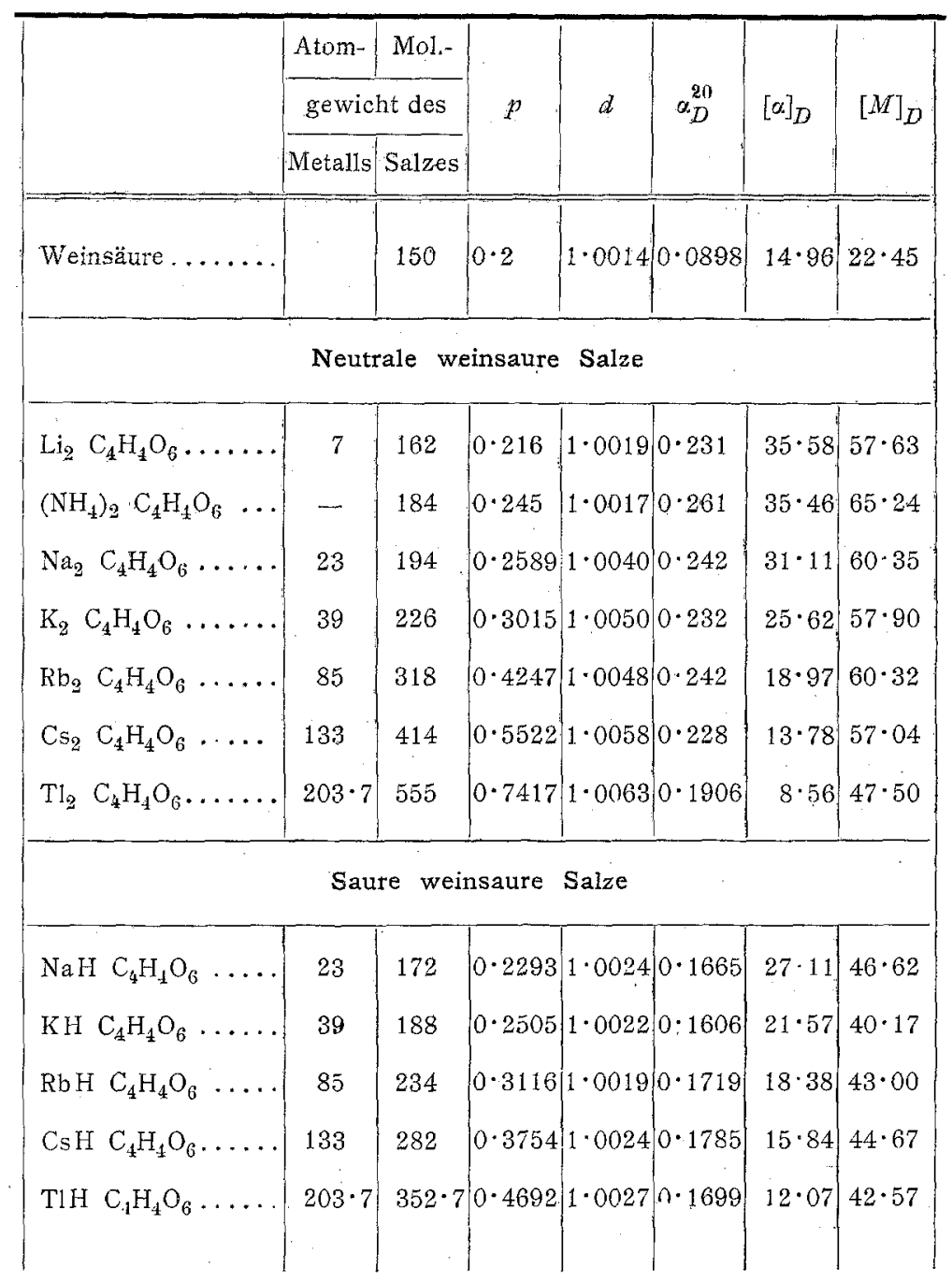

Sieht man zunächst von den berechneten Werthen ab und zieht man nur die direct beobachteten Drehungswinkel $\alpha_{D}^{20}$ in Betracht, so lässt sich nicht verkennen, dass dieselben bei den sauren Salzen als gleich angesehen werden können, und dass sie, mit Ausnahme des Thalliumtartrates auch bei den neutralen Salzen grosse Annäherung zeigen. 
Das neutrale Thalliumtartrat gibt aber eine Abweichung von dem mittleren beobachteten Drehungswinkel, die nicht mehr in den Fehlergrenzen des Apparates ihre Erklärung findet, da directe Versuche ergaben, dass die Beobachtungsfehler 0.02 nie erreichen.

Die Abweichung von der Oudemann'schen Regel, deren Richtigkeit durch alle übrigen Versuche bestätigt wird, könnte in dem einem Falle nur so erklärt werden, dass man den gewählten Concentrationsgrad als noch nicht verdünnt genug ansehen wollte, dass also die Dissocation noch keine vollkommene war. Lösungen, die weniger als $0 \cdot 2 \%$ Weinsäure enthaiten, dürften allerdings mit den bis jetzt zur Verfügung stehenden Apparaten schwer zu untersuchen sein.

Der Umstand, dass gerade dasjenige Tartrat, dessen Metallbestandtheil durch ein grosses Atomgewicht ausgezeichnet ist, die Ausnahme bildete, legte den Gedanken nahe, dass in Übereinstimmung mit der Ansicht von Guye das Drehungsvermögen der activen Substanz von dem Gewichte des nicht activen Theiles beeinflusst werden kann. Es war deshalb von Interesse die Lösungen des Thalliumtartrates in verschiedenen Concentrationen zu untersuchen.

Die Resultate gibt folgende Tabelle:

Tabelle II.

Neutrales weinsaures Thallium.

\begin{tabular}{|c|c|c|c|c|c|c|c|c|c|}
\hline Atom- & Mol.- & & & & & & & & \\
\hline gewic & ht des & $p$ & $d$ & $\alpha_{D}^{20}$ & {$[\alpha]_{D}$} & {$[M]_{D}$} & an & von $L$ & ong 1 \\
\hline Metalls & Salzes & & & & & & & $\alpha_{D}$ & {$[\alpha]_{D}$} \\
\hline $203 \cdot 7$ & 555 & 0.7417 & $1 \cdot 0063$ & $0 \cdot 1906$ & $8 \cdot 56$ & $47 \cdot 50$ & $0 \cdot 2$ & - & $\ldots$ \\
\hline $203 \cdot 7$ & 555 & $4 \cdot 9619$ & $1 \cdot 0421$ & $|0 \cdot 6801|$ & $4 \cdot 58$ & $25 \cdot 30$ & $1 \cdot 351$ & 0.667 & $4 \cdot 729$ \\
\hline $203 \cdot 7$ & 555 & $14 \cdot 060$ & $1 \cdot 1294$ & $1 \cdot 1291$ & $2 \cdot 68$ & $14 \cdot 87$ & $3 \cdot 845$ & - & - \\
\hline $203 \cdot 7$ & 555 & $27 \cdot 230$ & $1 \cdot 2294$ & $1 \cdot 6336$ & $2 \cdot 28$ & $12 \cdot 65$ & $7 \cdot 69$ & - & - \\
\hline
\end{tabular}

1 Die in den beiden letzten Reihen angeführten Zahlen sind Beobachtungen von Long entnommen. (Amer. Journ. of Sc., $3^{0}$ serie, t. 38 .) 
Weinsäure.

\begin{tabular}{|c|c|c|c|}
\hline$p$ & $\alpha_{D}^{20}$ & {$[\alpha]_{D}$} & {$[M]_{D}$} \\
\hline 0.2 & 0.089 & 14.96 & 22.45 \\
1.351 & 0.60 & 14.88 & 22.32 \\
3.845 & 1.67 & 14.56 & 21.84 \\
7.69 & 3.24 & 14.05 & 21.08 \\
& & &
\end{tabular}

Die moleculare Drehung $27 \%$-Lösung des neutralen Thalliumtartrates, welche $7 \cdot 69 \%$ Weinsäure enthält, beträgt $12 \cdot 65$, während Lando $1 \mathrm{t}^{1}$ für die moleculare Drehung der von ihm unitersuchten Tartrate bei gleichem Weinsäuregehalt im Mittel die Zahl 63 gefunden hat. Diese bedeutende Abweichung kann nur dadurch erklärt werden, dass es sich in dem vorliegenden Fall um ein Salz handelt, welches ein weit höheres Moleculargewicht als die von. Landolt untersuchten Salze besitzt und es zeigen diese Versuche, dass mindestens in concentrirteren Lösungen der Einfluss des Gewichtes des inactiven Bestandtheiles unverkennbar ist.

Guye $^{2}$ hat auf Grund der Beobachtung an 26 Tartraten darauf hingewiesen, dass die Lösung derselben durchwegs eine grössere Drehung zeigen als die einer Weinsäurelösung von gleichem Gehalt an Weinsäure, und unter Hinweis auf meine früheren Versuche, ${ }^{3}$ aus welchen hervorgeht, dass auch organische Basen die Drehung der Weinsäure erhöhen, sprach Guye die Ansicht aus, dass diese Eigenschaft allen Basen zukomme.

Es ist nun bemerkenswerth, dass, wie die vorliegenden Beobachtungen zeigen, das Thallium sich anders verhält, nndem es wohl in verdünnter Lösung die Drehung der Weinsäure erhöht, in concentrirterer dagegen dieselbe erheblich vermindert.

1 1. c. S. 1076 .

2 Archives des sciences physiques et naturelles Genève, t. 26, p. 338.

3 Sitzungsber. der kais. Akad. der Wissensch. in Wien, mathem.-naturw. Cl., 47, 460 (1888). 
Trägt man die für die Drehung des neutralen Thalliumtartrates und einer Weinsäurelösung von gleichem Gehalt an Weinsäure gewonnenen Zahlen ${ }^{1}$ in ein Coordinatennetz ein, so zeigt sich deutlich, dass die so erhaltenen beiden Curven einen Durchschnittspunkt haben, d. h. dass bei einer bestimmten Concentration das nicht active Thallium auf das Drehungsvermögen ohne Einfluss ist.

Ich enthalte mich vorläufig weiterer Schlussfolgerungen, hoffe aber, über die Fortsetzung dieser Versuche, welche auch auf andere active Säuren ausgedehnt werden, bald ausführlicher berichten zu können.

1 Die Zahlen für die Weinsäure sind theils den directen Beobachtungen von Landolt und Sonnenthal entnommen, theils aus der von Landolt aufgestellten Interpolationsformel berechnet. 